EDITORIAL

\title{
Educación superior, conocimiento y formación en Cirugía
}

\author{
José Félix Patiño RestrePo*
}

Palabras clave: educación médica; cirugía general; docentes médicos; rol del médico.

Como uno de los integrantes de la Comisión para la Reforma de la Educación Médica recientemente creada por los Ministerios de Salud y de Educación, se tiene la oportunidad de reflexionar más allá de las interesantes discusiones que tuvieron lugar en la Academia Nacional de Medicina, sede de las reuniones.

La educación en Cirugía, la de pregrado y la de posgrado, ha sido motivo de interés desde la época de Hipócrates hace dos mil quinientos años. En el Juramento Hipocrático ya se consignan valores fundamentales y, a lo largo de los años, se han planteado normas estrictas para la formación del cirujano.

En la formación del cirujano están implicados la educación superior, el conocimiento y la formación por competencias.

Universalmente, se entiende por educación superior la que sigue a la educación secundaria que en Colombia termina con el título de bachiller, y se imparte en las universidades y en los institutos politécnicos como formación profesional o formación académica, y se caracteriza por la investigación científica.

* Editor, Revista Colombiana de Cirugía

Fecha de recibido: 2 de junio de 2017

Fecha de aprobación: 6 de junio de 2017

Citar como: Patiño JF. Educación superior, conocimiento y formación en Cirugía. 2017;32:79-81.
En Colombia, la educación superior se inicia al terminar el bachillerato y es de tipo "profesionalista" más que académica. Al finalizar una carrera, el estudiante puede ingresar a la formación en una especialidad que, en el caso de la Medicina, se hace en los programas de residencia; en las otras carreras se realiza la especialización en programas que llevan a la maestría o al doctorado y también al posdoctorado. En Medicina, el título de especialista es equivalente al de maestría, como lo planteó la Ley 100 de 1993 en su Artículo 247:

“[...] Los programas de especializaciones médicoquirúrgicas que ofrezcan las instituciones universitarias y las universidades, tendrán un tratamiento equivalente a los programas de maestría, conforme a lo contemplado en la Ley 30 de 1992, previa reglamentación del Consejo de Educación Superior [...]”".

En la educación superior anglosajona, al término de la educación secundaria comienza la formación intelectual y cultural de la persona a través de los estudios generales o de educación liberal, que cubre las ciencias, las humanidades y las artes, con programas universitarios de cuatro años de duración. Es lo que se conoce como college.

La Asociación Colombiana de Facultades de Medicina (Ascofame) realizó un importante seminario, Estudios Básicos Generales en Educación Médica, en la ciudad de Buga en marzo de 1966, en plena época de la gran reforma de la educación médica colombiana que pasó del modelo anatomoclínico francés al modelo biológico y social estadounidense, y se concluyó que 
"[...] Los campos del conocimiento indispensables en el mundo actual para que cualquier profesional, y especialmente el médico, adquiera la formación necesaria que le permita desarrollar adecuadamente sus actividades, tanto las específicas de su profesión como las complementarias de su personalidad humana, comprenden: las humanidades, las matemáticas, las ciencias naturales o experimentales y las ciencias sociales. Son ellas verdaderos cimientos de la cultura y ninguna persona educada, mucho menos el médico, puede prescindir de ninguna de ellas $[\ldots]$ ". ${ }^{1}$.

Tres documentos establecen el fundamento de la educación superior:

1. El Ratio Studiorum de los jesuitas, fechado en 1598 y publicado oficialmente en 1599, plantea la sistematización, la organización y el método de los estudios en los colegios y universidades de la Compañía de Jesús. En ella se halla la concepción filosófica y pedagógica de la educación jesuítica.

2. El Yale Report de 1828 es uno de los documentos de mayor influencia en el desarrollo de la educación superior en los Estados Unidos. Consiste de dos partes: la primera parte, la más importante, es una discusión general sobre la naturaleza de la educación liberal. En nuestro medio, ésta se conoce como "estudios generales" y sólo la Universidad de los Andes, desde su fundación, los ha mantenido como parte integral de todos sus programas de educación superior. Fueron introducidos en la Universidad Nacional (y en otras universidades de Colombia) con la reforma de 1964-1966, como lo principal de la reforma académica, y para lo cual se integraron las tres grandes facultades que reúnen el conocimiento universal: la Facultad de Ciencias, la Facultad de Artes y la Facultad de Ciencias Humanas, pero luego desaparecieron en el curso de unos doce años. Esta reforma es conocida también como la "Reforma Patiño ${ }^{2}$. La segunda parte del Yale Report es de enfoque estrecho y específico, $\mathrm{y}$ actualmente se la incluye a efecto de presentarlo completo.

3. The Idea of a University ${ }^{3}$, el reconocido texto por el Cardenal John Henry Newman (1801-1890) de Oxford, que reúne las conferencias dictadas con ocasión del inicio de la Universidad Católica de Irlanda en Dublín, publicado en 1852. Plantea que la función principal de una universidad es la "educación liberal" (estudios generales), que es el lugar donde se accede al conocimiento universal, dirigido a formar intelectualmente a la persona, sin otro propósito que cultivar la inteligencia y el conocimiento como valor: "[...] La educación liberal es libre y entraña la formación del intelecto, para que busque en todo momento la verdad y la realice [...]".

La Epistemología es la rama de la Filosofía que estudia el conocimiento, que es el contenido intelectual pertinente a un área determinada o a la totalidad del universo; es el conjunto de información lograda mediante el aprendizaje, la investigación y la experiencia o a través de la introspección, entendida ésta, según la Word Reference, como la observación interna de los pensamientos, sentimientos o actos.

El conocimiento biomédico es el más amplio y el de más rápido recambio, de tal forma que lo que hoy es verdad en cinco o seis años ya no lo será. Ello es especialmente válido en Cirugía, que en su desarrollo se ha vuelto cada vez más dependiente de la tecnología, con el consiguiente peligro de la deshumanización. Pero, por una parte, mientras algunos sostienen que el desarrollo de la cirugía dependiente de la tecnología llevará a que el cirujano del futuro sea más bien un bioingeniero deshumanizado, por otra, muchos pensamos que la tecnología es humanizante en cuanto es una amplificadora de la capacidad intelectual y física del ser humano. El computador, la más maravillosa de las máquinas desarrolladas por el hombre, no es sino un amplificador de la capacidad intelectual y un servidor del conocimiento, por lo cual hemos planteado que no se lo vea solo a la luz de la informática, entendida como la ciencia y la tecnología del manejo de la información, sino en términos de la metafísica de la informática ${ }^{4}$.

La "teoría médica formal" y la creciente dimensión del conocimiento biomédico y su aplicación racional de la tecnología de punta son las fuerzas que determinan la práctica quirúrgica.

La educación médica actual se fundamenta en planes de estudio organizados por asignaturas con enfoque por competencias; el concepto de competencia se entiende de manera amplia como "saber hacer en contexto". Un ejemplo es el adoptado en los últimos años por la Universidad Nacional Autónoma de México ${ }^{5}$. Tales planes vienen siendo implementados desde la recomendación de la Conferencia Mundial sobre Educación Superior 
realizada por UNESCO en 1998, con resultados variables según el área del conocimiento. Un excelente artículo de revisión aparece en la Revista Médica del Hospital General de México ${ }^{6}$. La educación por competencias ha sido implementada en Colombia, en los últimos años, por el Ministerio de Educación Nacional, con la competencia como el principio organizador del currículo. Diversas instituciones y las universidades organizan sus currículos por competencias, desde las que definió el Institute for International Medical Education como estándares mínimos para las facultades de Medicina ${ }^{7}$, hasta la enseñanza de la Cirugía basada en competencias de la Universidad de Buenos Aires ${ }^{8}$.

Finalmente, es pertinente referirse a Sir Peter Medawar (1915-1987), premio Nobel de Medicina o Fisiología
(1960), con quien tuve la oportunidad de trabajar durante un tiempo que él pasó en la Universidad de Yale donde yo estudiaba Medicina. En su libro The threat and the glory. Reflections on science and scientists (Oxford University Press) ${ }^{9}$, Medawar plantea que hay dos clases de pensamiento: pensamiento duro y pensamiento blando. El primero transmite un significado preciso, plantea ideas que pueden ser comprobadas por el método científico, que merecen análisis crítico y que despiertan inquietud intelectual. El segundo, el pensamiento blando, hace planteamientos atractivos a través de las emociones (lo que llamamos demagogia) y trata de persuadir sobre supuestas verdades intelectuales por métodos no intelectuales.

Ciertamente, en Cirugía sólo cabe el pensamiento duro.

\section{Referencias}

1. Moreno B, Castellanos J, editores. Educación médica, factor en el desarrollo económico y social. Estudios básicos generales en educación médica. Conclusiones y recomendaciones de la V Asamblea General. Bogotá: Ascofame; 1966.

2. Magnusson WE. La reforma Patiño UN 1964-1966. Una experiencia de construcción institucional. Bogotá: Unibiblos; 2006.

3. Newman JH. The idea of a University. New York: Yale University Press; 1966.

4. Patiño Restrepo JF. ¿La tecnología amenaza la relación médicopaciente? Rev Colomb Cir. 2012;27:97-8.

5. Sánchez-Mendiola M, Durante-Montiel I, Morales-López S, Lozano-Sánchez R, Martínez-González A, Graue-Wiechers E. Plan de estudios 2010 de la Facultad de Medicina de la Universidad Nacional Autónoma de México. Gaceta Méd México. 2011;147:152-8.

6. García-García JA, González-Martínez JF, Estrada-Aguilar L, Uriega-González S. Educación médica basada en competencias. Rev Med Hosp Gen Mex. 2010;73:57-69.
7. Institute for International Medical Education, Core Committee. Global minimum essential requirements in medical education. Medical Teacher. 2002;24:125-9.

8. Montesinos MR. Enseñanza de la cirugía basada en competencias en el pregrado de medicina. Rev Argent Cirugía. 2013;104:77-85.

9. Medawar PB. La amenaza y la gloria: reflexiones sobre la ciencia y los científicos. Barcelona: Editorial Gedisa; 1993.

Correspondencia: José Félix Patiño, MD

Correo electrónico: jfpatinore@gmail.com

Bogotá, D.C. 